\title{
Evolution of strength and coercivity during annealing of FeCo based alloys.
}

\author{
T. Sourmail \\ Department of Materials Science and Metallurgy, University of Cambridge \\ Pembroke Street, Cambridge CB2 3QZ, U.K.
}

Scripta Mater. 51:2004, 589-591

\begin{abstract}
Iron-cobalt alloys are used as soft-magnetic materials in circumstances where a high magnetic saturation is important. There is confusion in the literature about the cause of an observed discontinuity in the variation of strength and coercivity as a function of the temperature at which cold-rolled Fe-Co alloys are annealed. It is shown here using calculations based on published data, that the variety of observations can be reconciled on the basis of grain growth rather than the extent of recrystallisation.
\end{abstract}

\section{Introduction}

FeCo alloys have for some time been the materials of choice for applications where a high magnetic saturation is a prime design parameter, particularly in the aerospace industries where volume and mass need to be minimised.

There has been a resurgance of interest in these alloys, particularly with the development of very strong variants $[1,2]$. The interest is driven primarily by the need for a soft-magnetic material for the construction of electric motors which can be embedded in hot regions of aircraft engines $[3,4,5]$. In such applications, the alloys are required in a laminated form in order to reduce 
eddy current losses. The production process therefore involves cold-rolling, followed by annealing in dry $\mathrm{H}_{2}$ at temperatures in the range 720 and $850{ }^{\circ} \mathrm{C}$.

In the following, all compositions indicate weight percent added to an equiatomic FeCo alloy (or near equiatomic as most publications do not specify).

There is disagreement in the literature over the conditions required to achieve full recrystallisation. Thornburg [1] studied a FeCo-2\%V alloy cold rolled to $90 \%$ reduction in thickness, then annealed $2 \mathrm{~h}$ at temperatures in the range 640 to $760{ }^{\circ} \mathrm{C}$ followed by air cooling to room temperature. It was found that the strength and coercivity decreased sharply as the annealing temperature was increased, until about $710{ }^{\circ} \mathrm{C}$, beyond which the properties changed little (see FeCo-2V in figure 1).

Hailer [6] repeated the experiments on FeCo-2V-0.3Nb and obtained similar results: both strength and coercivity decreased sharply until an annealing temperature of about $730{ }^{\circ} \mathrm{C}$ (figure 1 ). In both cases, the authors attributed the observations to incomplete recrystallisation below these temperatures. Unfortunately, neither measured the grain size as a function of annealing temperature, as a matter of fact, neither undertook detailed metallography of the material.

Although seemingly reasonable, the interpretation in terms of the extent of recrystallisation contradicts a number of independent results. For example, Buckley [7] observed full recrystallisation of cold-rolled FeCo-0.4Cr at low temperatures $\left(250-475^{\circ} \mathrm{C}\right)$ and suggested that, in this range of temperatures, recrystallisation was enhanced by the ordering reaction typical in FeCo alloys. Davies and Stoloff also reported recrystallisation of FeCo-2V after only $1 \mathrm{~h}$ at $675^{\circ} \mathrm{C}$; more recently, Duckham et al. [2] obtained recrystallised structures after $1 \mathrm{~h}$ at $650{ }^{\circ} \mathrm{C}$ in $\mathrm{FeCo}-2 \mathrm{~V}-0.3 \mathrm{Nb}$. Unfortunately, none of these authors reported the variations of strength or coercivity with annealing temperature, at least not over a range of temperature large enough for comparison with 
Thornburg [1] or Hailer [6].

In the present work, published data for grain growth, coercivity and strength are used to solve the apparent inconsistencies.

\section{Strength, coercivity and grain growth}

An exhaustive review of studies undertaken on magnetic and mechanical properties of FeCo based alloys has recently been completed [8]. By gathering a vast amount of published data, it was shown that the coercivity of most existing FeCo based alloys (FeCo, FeCo-V, FeCo-V-Nb, FeCo-Ta), in the annealed condition, is dictated essentially by the grain size, and that a unique line can be used to approximate the dependence:

$$
H_{c}=36.7+777.5 \times d^{-1}
$$

where $H_{c}$ is the coercivity in $\mathrm{A} \mathrm{m}^{-1}$, and $d$ the grain size in $\mu \mathrm{m}$.

A number of studies have reported the influence of grain size on strength (for example, $[9,10,11,12]$ ) and interpreted the results in terms of a HallPetch relationship:

$$
\sigma_{y}=\sigma_{0}+k d^{-1 / 2}
$$

where $\sigma_{y}$ is the strength, $\sigma_{0}$ a constant related to the intrinsic resistance to dislocation motion, and $k$ a constant related to the difficulty of activating dislocation sources in adjacent grains [13].

Although the parameters reported in these publications seem to differ significantly, it can be shown [8] that they are roughly equivalent over the range of grain sizes usually observed. These parameters are reported in table 1.

In the analysis that follows, the parameters reported by Jordan and Stoloff [12] are used, so that:

$$
\sigma_{y, \text { ord }}=143+714 \times d^{-1 / 2} \quad \mathrm{MPa}
$$




\begin{tabular}{|c|c|c|c|c|}
\hline \multirow[t]{2}{*}{ Composition } & Peierls stress & $k$ & Method & \multirow[t]{2}{*}{ Reference } \\
\hline & / $\mathrm{MPa}$ & $\mathrm{MPa} \mu \mathrm{m}^{-1}$ & & \\
\hline \multicolumn{5}{|l|}{$\mathrm{FeCo}$} \\
\hline ordered & 172 & 806 & compression & {$[11]$} \\
\hline disordered & 309 & 456 & tests & \\
\hline \multicolumn{5}{|l|}{$\mathrm{FeCo}-2 \mathrm{~V}$} \\
\hline ordered & 143 & 714 & tensile & {$[12]$} \\
\hline disordered & 435 & 242 & tests & \\
\hline $\begin{array}{l}\text { FeCo-2V } \\
\text { ordered }\end{array}$ & 26 & 848 & tensile tests & {$[10]$} \\
\hline
\end{tabular}

Table 1: Different values of $\sigma_{0}$ and $k$ for ordered and disordered samples of FeCo based alloys.

for ordered alloys, and

$$
\sigma_{y, \text { disord }}=435+242 \times d^{-1 / 2} \quad \mathrm{MPa}
$$

for disordered alloys.

Few studies have looked at grain growth in FeCo based alloys. It appears that only Davies and Stoloff [14] provide the data required to understand the impact of heat-treatment on strength and coercivity. However, neither Thornburg nor Hailer seem to have been aware of this study.

Davies and Stoloff [14] studied the kinetics of grain growth at temperatures ranging from 675 to $775{ }^{\circ} \mathrm{C}$ and reported significantly different behaviour above and below the critical temperature for ordering $T_{c} \sim 720 \mathrm{deg}$. In particular, while grain growth was reasonably fast above $T_{c}$, it was found to be much slower below this temperature. Table 2 lists the grain sizes after $1 \mathrm{~h}$ annealing. 


\begin{tabular}{cc}
\hline Annealing temperature $/{ }^{\circ} \mathrm{C}$ & Grain size $/ \mu \mathrm{m}$ \\
\hline 675 & 0.6 \\
688 & 0.8 \\
700 & 1.4 \\
711 & 3.0 \\
725 & 7.9 \\
750 & 12.0 \\
$775^{*}$ & 17.1 \\
\hline
\end{tabular}

Table 2: The grain size of an FeCo-2V alloy annealed $1 \mathrm{~h}$ at different temperatures, data extracted from [14]. * indicates a point which was not measured but extrapolated, the last point for this temperature being for 45 min annealing.

\section{Calculated strength, coercivity and com- parison}

From the grain sizes reported in table 2, the expected strength and coercivity of the alloy studied by Davies and Stoloff were calculated [14]. Both Thornburg and Hailer cooled their samples in air after annealing. Given the kinetics of the ordering reaction in FeCo based alloys [8], it is difficult to ascertain the final degree of order in these samples, which was not measured by the authors.

For this reason, the strength of FeCo alloys was at first calculated using equation 3 for all annealing temperatures, and then, using equation 3 for annealing temperatures below $T_{c}$ and equation 4 for annealing temperatures above $T_{c}$. The results are shown in figures 1 and 2 .

The agreement between the calculated and measured values is surprisingly good, as it must be remembered that equations 2, 3 and 4 have been derived using different experimental data, obtained using annealing temperatures greater than $T_{c}$. 


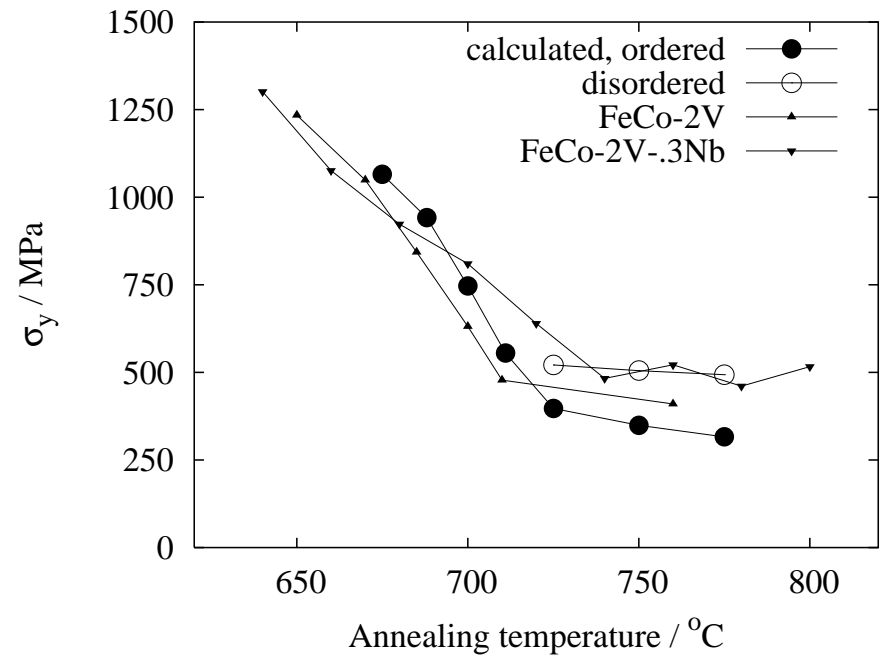

Figure 1: The strength of FeCo-V and FeCo-V-Nb alloys, as measured by Thornburg [1] and Hailer [6] respectively. Calculation for FeCo-2V using the grain sizes reported by Davies and Stoloff [14], assuming in a first instance, that all samples are ordered (filled circles), and in a second study, that samples cooled from above $T_{c}$ are disordered (empty circles).

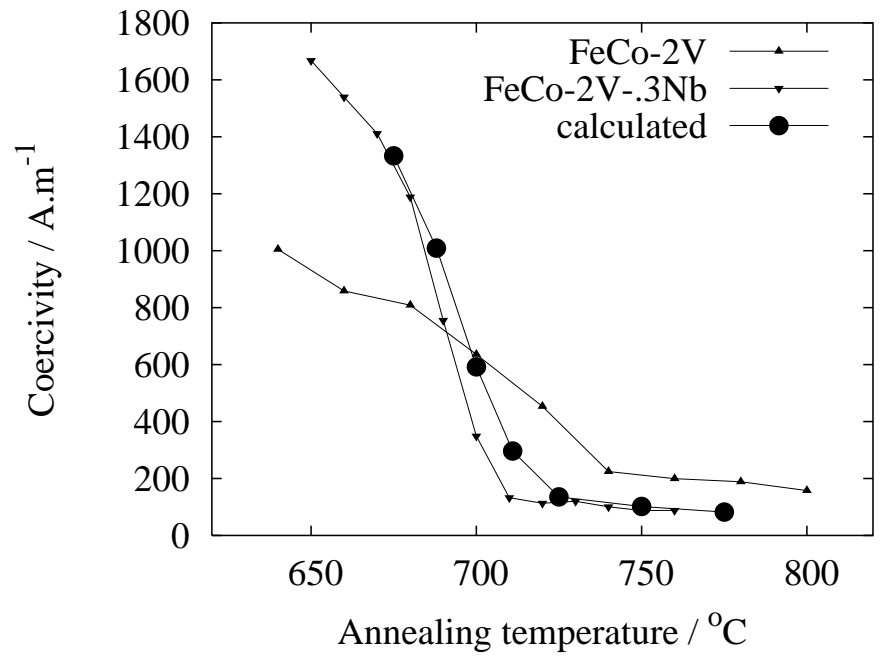

Figure 2: The coercivity of FeCo- $\mathrm{V}$ and FeCo-V-Nb alloys, as measured by Thornburg [1] and Hailer [6] respectively. Calculation for FeCo-2V using equation 1 and the grain sizes reported by Davies and Stoloff [14]. 
The important result is that all the observed variations can be explained quantitatively on the basis of grain size variations alone, rather than by postulating variations in the extent of recrystallisation.

\section{Summary and conclusions}

Using published data on magnetic and mechanical properties of FeCo based alloys, it has been possible to show that the coercivity and the strength of FeCo-2V as a function of annealing treatment can be fully understood in terms of the fully recrystallised grain size.

The sharp change in strength and coercivity versus annealing temperature around $720^{\circ} \mathrm{C}$ has in the past been attributed to incomplete/complete recrystallisation $[1,6]$. The present analysis shows that the changes are fully understood in terms of the grain size of fully recrystallised samples. The reason for the discontinuity in the property versus annealing temperature curves is simply that rate of grain growth changes sharply around critical ordering temperature $T_{c}$.

\section{Acknowledgements}

The author is grateful to Rolls-Royce, in particular S. W. Hill, for funding the project this work is part of, and to Pr. H. K. D. H. Bhadeshia for useful discussion.

\section{References}

[1] D. R. Thornburg, J. Appl. Phys. 40 (1969) 1579-1580.

[2] A. Duckham, D. Z. Zhang, D. Liang, V. Luzin, R. C. Cammarata, C. L. Leheny, C. L. Chien, T. P. Weihs, Acta Mater. 51 (2003) 4083-4093. 
[3] R. T. Fingers, R. P. Carr, Z. Turgut, J. Appl. Phys. 91 (10) (2002) $7848-7850$.

[4] R. H. Yu, S. Basu, R. Y. Zhang, A. Parvizi-Marjidi, K. M. Unruh, J. Q. Xiao, IEEE Trans. Magn. 36 (5) (2000) 3388-3393.

[5] B. Geist, T. Peterson, J. C. Horwath, Z. Turgut, M. Q. Huang, R. A. Snyder, R. T. Fingers, J. Appl. Phys. 93 (10) (2003) 6686-6688.

[6] B. T. Hailer, Master's thesis, Virginia Polytechnic Institute, Blacksburg, Virginia, http://scholar.lib.vt.edu/theses/available/etd-05032002$144727 /(2001)$.

[7] R. A. Buckley, Metal Sci. 13 (1979) 67-72.

[8] T. Sourmail, H. K. D. H. Bhadeshia, Microstructure, magnetic and mechanical properties of FeCo based alloys, Tech. rep., Advanced Aerospace Materials DARP, Rolls-Royce (2004).

[9] C. H. Shang, R. C. Cammarata, T. P. Weihs, C. L. Chien, J. Mater. Res. 15 (4) (2000) 835-837.

[10] L. Ren, S. Basu, R. H. Yu, J. Q. Xiao, A. Parvizi-Majidi, J. Mater. Sci. 36 (2001) 1451-1457.

[11] L. Zhao, I. Baker, Acta Metall. Mater. 42 (6) (1994) 1953-1958.

[12] K. R. Jordan, N. S. Stoloff, Trans. Metal. Soc. AIME 245 (1969) 20272034.

[13] V. Bata, E. V. Pereloma, Acta Mater. 52 (2004) 657-665.

[14] R. G. Davies, N. S. Stoloff, Trans. Met. Soc. AIME 236 (1966) 16051608. 